\title{
Robotic Microsurgery Optimization
}

\author{
Jamin V Brahmbhatt ${ }^{1}$, Ahmet Gudeloglu ${ }^{2}$, Philippe Liverneaux ${ }^{3}$, Sijo J Parekattil ${ }^{1}$ \\ ${ }^{1}$ Personalized Urology \& Robotics, The PUR Clinic, South Lake Hospital, Clermont, FL, USA; ${ }^{2}$ Personalized Urology \& Robotics, The PUR \\ Clinic, Memorial Ankara Hospital, Ankara, Turkey; ${ }^{3}$ Department of Hand Surgery, Strasbourg University Hospitals, Strasbourg, France
}

The increased application of the da Vinci robotic platform (Intuitive Surgical Inc.) for microsurgery has led to the development of new adjunctive surgical instrumentation. In microsurgery, the robotic platform can provide high definition $12 \times-15 \times$ digital magnification, broader range of motion, fine instrument handling with decreased tremor, reduced surgeon fatigue, and improved surgical productivity. This paper presents novel adjunctive tools that provide enhanced optical magnification, micro-Doppler sensing of vessels down to a 1-mm size, vein mapping capabilities, hydro-dissection, micro-ablation technology (with minimal thermal spread- $\mathrm{CO}_{2}$ laser technology), and confocal microscopy to provide imaging at a cellular level. Microsurgical outcomes from the use of these tools in the management of patients with infertility and chronic groin and testicular pain are reviewed. All these instruments have been adapted for the robotic console and enhance the robotassisted microsurgery experience. As the popularity of robot-assisted microsurgery grows, so will its breadth of instrumentation.

Keywords Robotics / Microsurgery / Instrumentation / Equipment and supplies
Correspondence: Sijo J Parekattil Personalized Urology \& Robotics, The PUR Clinic, South Lake Hospital, 1900 Don Wickham Drive, Clermont, FL 34711, USA

Tel: $+1-352-536-8761$

Fax: +1-321-843-2120

Email: sijo_p@hotmail.com

\section{INTRODUCTION}

From the early beginnings of microsurgery, development of instrumentation to assist the microsurgeon in complex microsurgical procedures has been ongoing. The applicability of the robotic platform for microsurgery is improving due to the development of new microsurgery-specific robotic instrumentation. This article will focus on currently available tools and their optimization for robot-assisted microsurgery.

\section{ROBOTIC PLATFORM}

Since the first robot-assisted surgery in 1997 by Jacques Himpens and Guy Cardiere in Belgium, the technology has evolved and changed the landscape of modern day surgery. In micro- surgery, the 4-arm da Vinci robotic platform (Intuitive Surgical Inc., Sunnyvale, CA, USA) comes with the ability to provide high definition visual $12 \times-15 \times$ magnification, broader range of motion, fine instrument handling with decreased tremor, reduced surgeon fatigue, and improved surgical productivity [1]. As the popularity of robotic assisted microsurgery grows, so will the development of adjunctive instrumentation.

\section{ENDOWRIST ROBOTIC INSTRU- MENT}

The robotic instruments currently being utilized were initially designed for microsurgical applications in cardiac surgery. The most commonly used instruments in our microsurgical practice are: Black Diamond Micro Forceps, bipolar microforceps, Potts 
scissors, and monopolar curved scissors (Intuitive Surgical Inc.)

(Fig. 1).

The instruments are passed through $8-\mathrm{mm}$ trocars, even though the procedure is performed outside the body, to allow the instruments to function with stability. It is important to advance the instruments 4 to $5 \mathrm{~cm}$ beyond the tip of the trocar to optimize the range of motion. The Black Diamond Micro Forceps are used for retraction, suturing, micro-dissection, and

\section{Fig. 1. Instrumentation for microsurgical cases}

EndoWrist (Intuitive Surgical Inc.) instrumentation for robotic microsurgical cases. The bipolar forceps can be used for cautery, retraction, or suturing. The Black Diamond Micro Forceps can be used for fine dissection, retraction, and suturing.

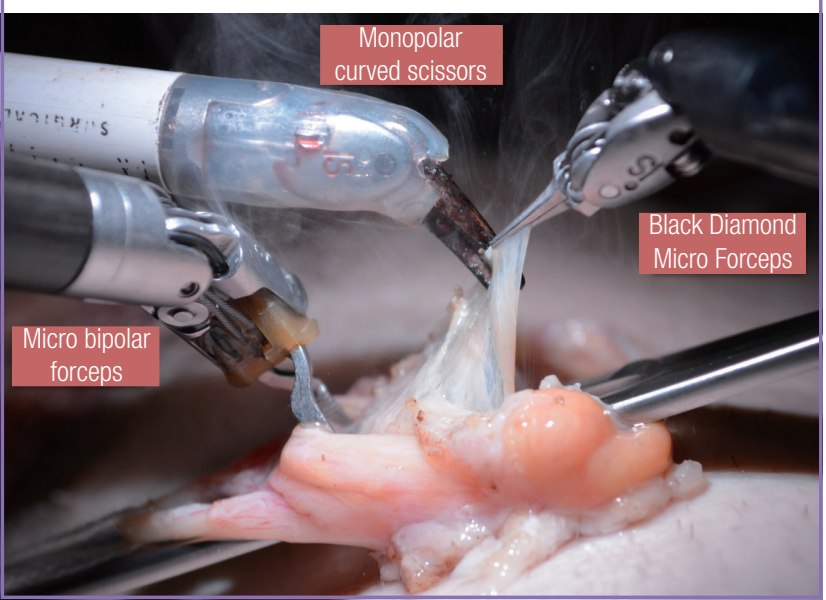

holding adjunctive microsurgical instruments discussed later in this paper. The bipolar microforceps assist with fine cauterization, suturing, and holding adjunctive microsurgical instruments discussed later in this paper.

\section{TELESCOPIC MAGNIFICATION}

Microsurgical procedures require magnification of small structures. The increased pixilation that comes with digital $(12 \times-15 x)$ magnification on the robotic platform may not provide optimum visualization for microsurgical procedures. The telescope-based high definition video system (VITOM, Karl Storz, Tuttlingen, Germany) offers $16 \times-18 \times$ optical magnification comparable to the operating microscope. Recently, it has even been proposed as a viable alternative for microneurosurgery [2]. For robotic microsurgery, it can provide adjunctive imaging capabilities beyond the $12 \times-15 \times$ digital magnification of the robotic platform. The VITOM can be integrated into the robotic TilePro system (Intuitive Surgical Inc.) that provides the microsurgeon with a cockpit view of all video inputs (Fig. 2). In our practice the VITOM is positioned on an additional fifth robotic (nitrogen powered, Karl Storz) arm (Fig. 3).

We routinely use the VITOM for robot-assisted vasovasostomy (RAVV) and vasoepididymostomy (RAVE). Between July 2007 and March 2013, 147 robot-assisted vasectomy reversals (90 bilateral RAVV, 57 RAVE) were performed using

\section{Fig. 2. Cockpit view of robotic console}

(A) View with robotic $(10 x-15 x)$ magnification. (B) Shows the same image through a higher magnification VITOM lens $(16 \times-20 \times)$. (C) Real-time microscopic seen analysis $(40 x)$.
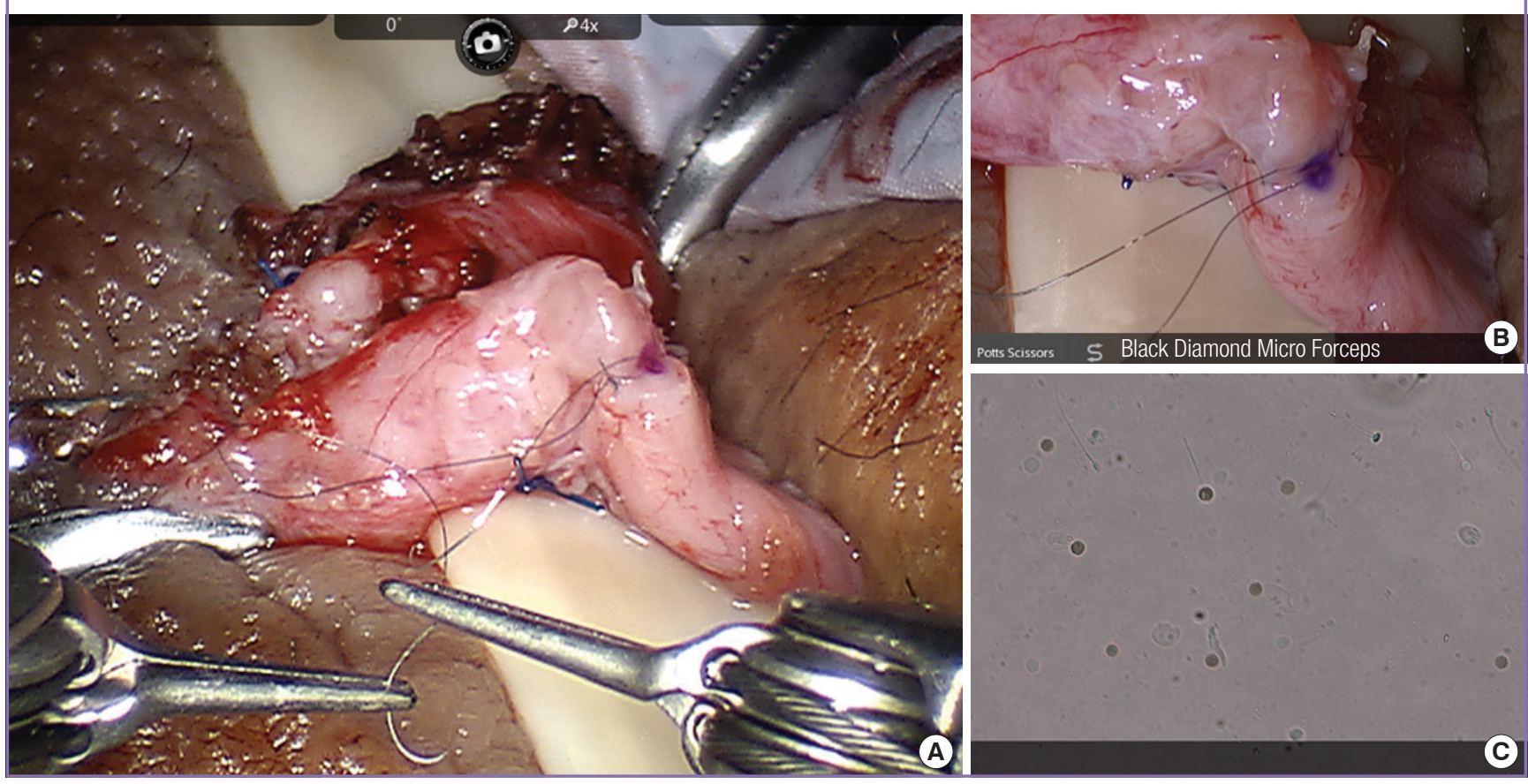


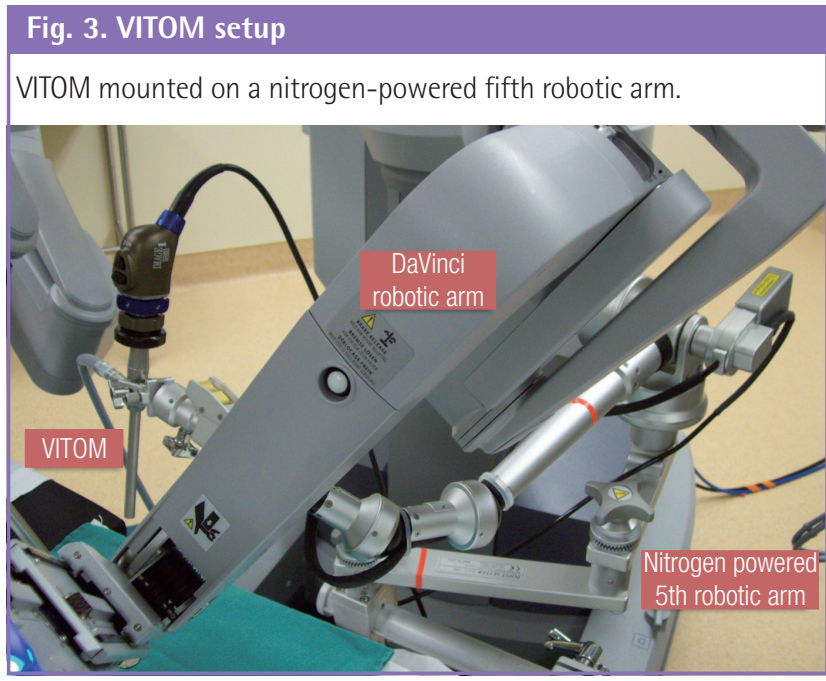

the VITOM. The median patient age was 42 years. 20 of these patients had chronic scrotal pain and the rest wished to regain their fertility. The median time period from vasectomy was 7 years for RAVE and 11 years for RAVE. At a median follow-up of 23 months, men who underwent RAVV and RAVE procedures had a patency rate ( $>1$ million motile sperm/ejaculate) of $97 \%$ and $60 \%$, respectively. There was significant pain relief in $88 \%$ of the patients who underwent a reversal for post-vasectomy pain. These results are comparable to those of traditional microscopic reversal. The use of robotic assistance with enhanced optical visualization with the VITOM for microsurgical vasectomy reversal may decrease the duration of the operation and significantly improve early semen analysis measures. Further evaluation and longer follow-up is needed to assess its clinical potential and the cost-benefit ratio.

\section{MICRODOPPLER}

Real time intraoperative Doppler ultrasound use during microsurgical procedures prevents arterial injuries and improves surgical efficiency $[3,4]$. Standard microscopic Doppler probes (MDP) did not conform to the robotic platform and required the use of a skilled surgical assistant. The flexible drop-in Doppler (Vascular Technology Inc., Nashua, NH, USA) was designed specifically for use with the robotic platform and allows easy manipulation by robotic forceps (Fig. 4). The surgeon can perform real-time Doppler monitoring of the surrounding vasculature and tissue. For microsurgical procedures, the $20-\mathrm{MHz}$ probe can be used for robot-assisted microscopic denervation of the spermatic cord, varicocelectomy, ligation of intracranial aneurysms, and nerve repairs. An alternate 8-MHz flexible drop-in probe is also available for tissues requiring a higher depth of penetration.

The flexible drop-in MDP was evaluated in 8 robotic micro-

\section{Fig. 4. Flexible drop-in micro-Doppler probe}

Robot-controlled Doppler probe used to identify testicular arteries and veins during denervation of the spermatic cord.

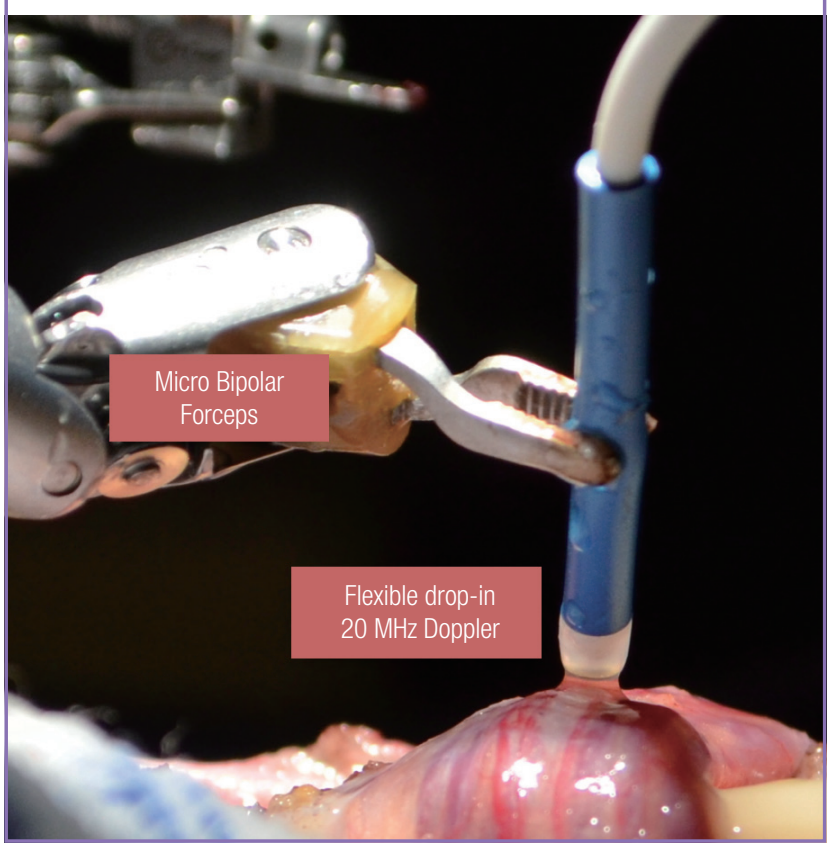

surgical cases ( 3 subinguinal varicocelectomies and 5 spermatic cord denervation procedures) for efficacy in testicular artery localization and ease of robotic grasper maneuverability. It was effective in identifying all of the testicular arteries within the spermatic cord in all cases. Due to the compact size of the MDP, maneuverability using the robotic grasper was significantly improved over the standard handheld Doppler probe. MDP allowed for a full range of motion of the robotic arms allowing the surgeon to easily scan vessels from a wide range of angles without the need for a skilled surgical assistant.

\section{MICRO-ULTRASOUND}

A robotically controlled ultrasound transducer (Aloka Inc., Wallingford, CT, USA) has recently been developed that allows surgeons to obtain full ultrasound imaging with real-time flow mapping [5]. Using the robotic graspers to manipulate this probe provides the surgeon with a full range of motion, articulation, and precision when scanning delicate organs (Fig. 5). The use of this probe also negates the need for a skilled surgical assistant. The probe is currently being modified for robot-assisted microsurgery.

\section{HYDRO-JET DISSECTION}

Hydro-jet dissection (HD) utilizes a thin, high-pressure stream of 


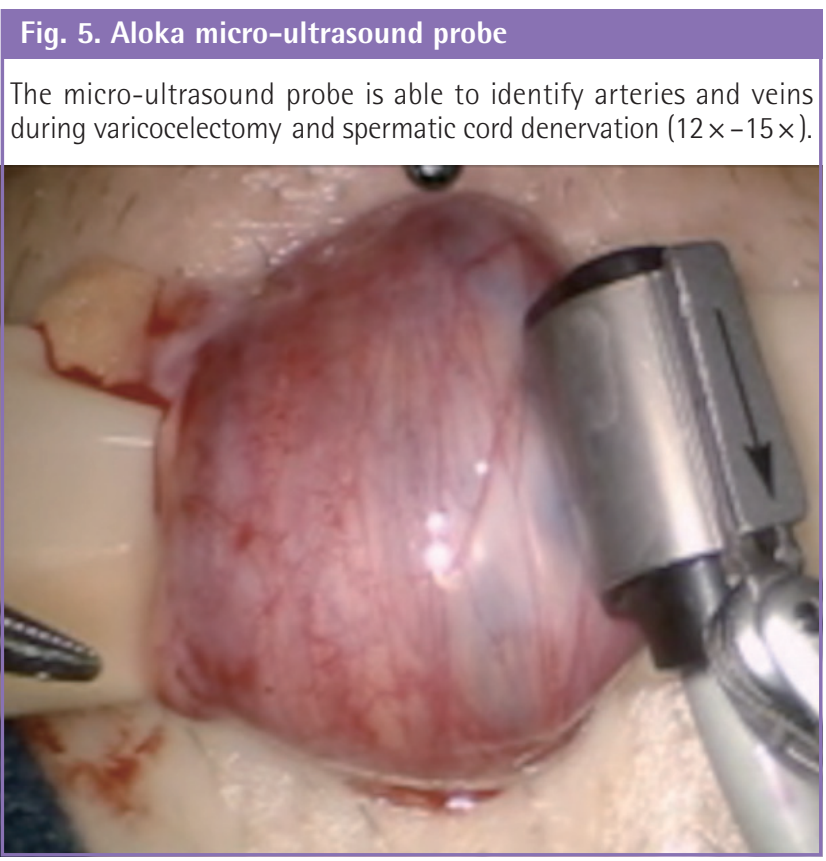

water for fine tissue dissection while preserving vascular integrity (Fig. 6). HD can be applied manually or with a pressure-adjustable device such as the ERBEJET2 hydrodissector (ERBE Inc., Tuebingen, Germany). The technology has been successfully applied during open and laparoscopic partial nephrectomy, retroperitoneal lymphadenectomy, and liver resection. Recently, it has been used for fine microsurgical tissue dissection in neurosurgical [6] and urologic procedures. Varying pressure settings can selectively destroy nerve fibers while preserving vascular tissue [7].

There are three primary locations of nerves within the spermatic cord: cremasteric muscle fibers, perivasal tissue and sheath, and posterior peri-arterial/lipomatous tissue [8]. Failures in microsurgical denervation of the spermatic cord (MDSC) can be due to residual small-diameter nerve fibers $(\leq 1 \mathrm{~mm})$ in these locations. A prospective randomized controlled trial was performed on 22 adult rats undergoing bilateral MDSC. HD was performed on one randomized side of the rat cord using $87 \mathrm{psi}$. The contralateral cord was used as a control (no HD). Cross sections of both cords were examined by a pathologist (blinded). In this study, the HD cords had a lower median residual nerve count compared to the non-HD side, from 8 to 5 nerves $(P=0.007)$. These findings show that HD can successfully ablate small-diameter nerve fibers. This could potentially then translate into improved outcomes after MDSC.

\section{$\mathrm{CO}_{2}$ LASER DISSECTION}

There are many options for ligation or dissection in microsurgery. Monopolar or bipolar cautery continue to be the gold standards
Fig. 6. ERBEJET2 hydrodissector for dissection of residual nerves

Hydrodissection of residual perivasal nerves during denervation of the spermatic cord.

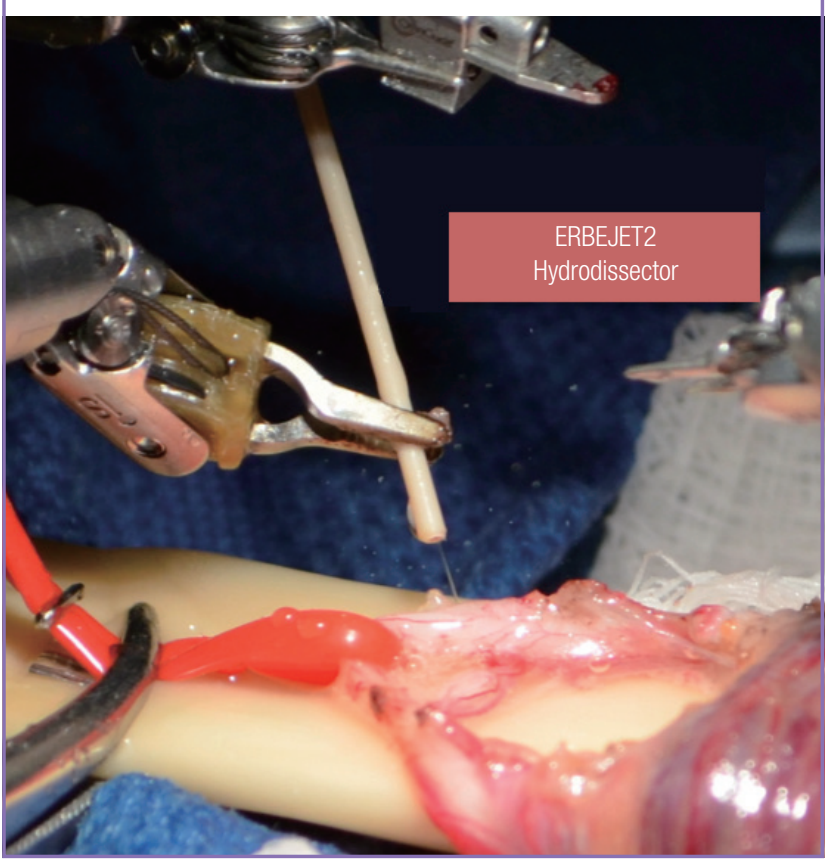

even though they can cause significant collateral tissue damage. The $\mathrm{CO}_{2}$ laser is now gaining popularity, given its ability to provide effective ligation and coagulation with less collateral thermal damage [9]. The laser is rapidly absorbed by the water in tissues, thus ensuring minimal thermal damage and spread. This property makes the $\mathrm{CO}_{2}$ laser well suited for dissection and ligation in microsurgery. A bulky delivery system and inability to resect in the microsurgeon's line of dissection previously limited its use in microsurgery. With the advent of the photonic band gap fiber assembly, a flexible fiber-optic $\mathrm{CO}_{2}$ delivery system was developed. The fiber is now integrated into a robotic hand piece (Fig. 7) that is manipulated with robotic arms (FlexGuide ULTRA and BeamPath Robotic Fiber, OmniGuide, Cambridge, MA, USA).

The fiber has been used for robotic-assisted myomectomy [10] for years and recently has been used for microsurgical procedures in otolaryngology [11]. The fiber-optic $\mathrm{CO}_{2}$ fiber was recently compared with the standard cautery for cadaveric vas deferens. Robotic MDSC with the $\mathrm{CO}_{2}$ laser was performed on one spermatic cord side and standard monopolar electrocautery was used on the contralateral side (control). Nine cross sections of the spermatic cord from each side were evaluated by a pathologist (blinded to technique and procedure). On microscopic pathology evaluation, the mean peripheral thermal damage in the spermatic cord was $0.17 \mathrm{~mm}$ (range, $0.15-0.25 \mathrm{~mm}$ ) on the side performed with $\mathrm{CO}_{2}$ laser ablation, significantly less compared to 
the contralateral monopolar cautery side with $0.72 \mathrm{~mm}$ (range, $0.60-0.75 \mathrm{~mm})(\mathrm{P}<0.001)$. There was no vascular or vasal trauma evident in either technique. The fiber-optic $\mathrm{CO}_{2}$ laser is a promising alternative to monopolar or bipolar cautery for microsurgical dissection, where it is important to maintain the integrity of the surrounding structures. Studies on the use of this laser for microdissection of the spermatic cord during denervation (for patients with chronic testicular or groin pain) are underway and appear promising.

\section{VEIN VIEWER}

The VeinViewer (Christie Digital Systems, Cypress, CA, USA) projects near-infrared light that is absorbed by the blood but reflected by surrounding tissue [12]. This information is captured, processed, and projected on the patient's body with a digital

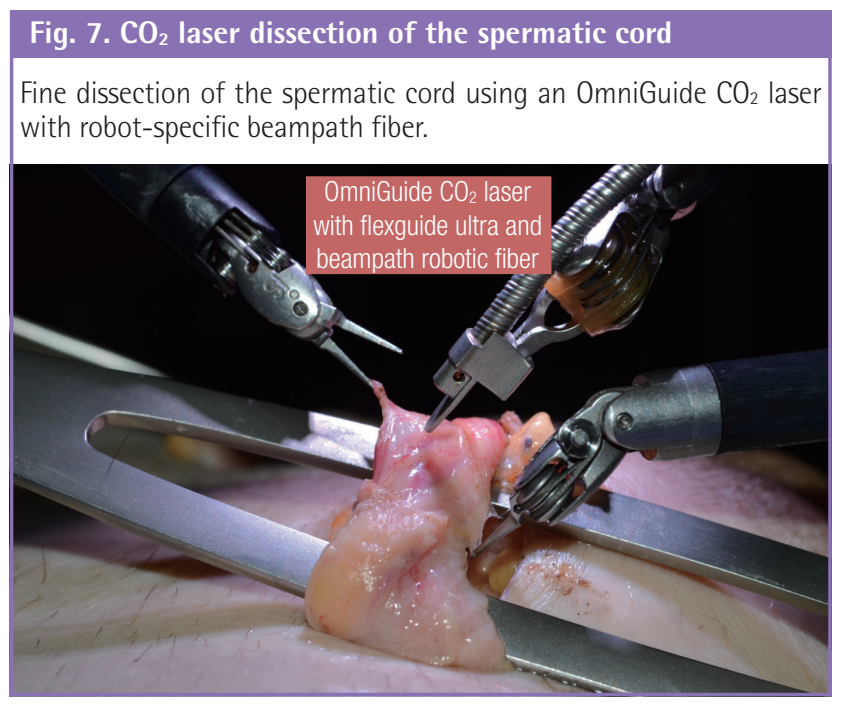

image of the patient's blood pattern. The technology is used for assistance with IV placement or dialysis access. The VeinViewer led to significantly greater likelihood of line placement in infants [13]. This same technology has recently been adopted for microsurgical varicocelectomy for the identification of small diameter veins. The viewer can be positioned over the spermatic cord and veins can be identified in real time (Fig. 8). The technology can be integrated with MDP to enhance the identification of key vascular structures.

\section{CONFOCAL MICROSCOPY}

The future of microsurgery appears to be imaging at the cellular

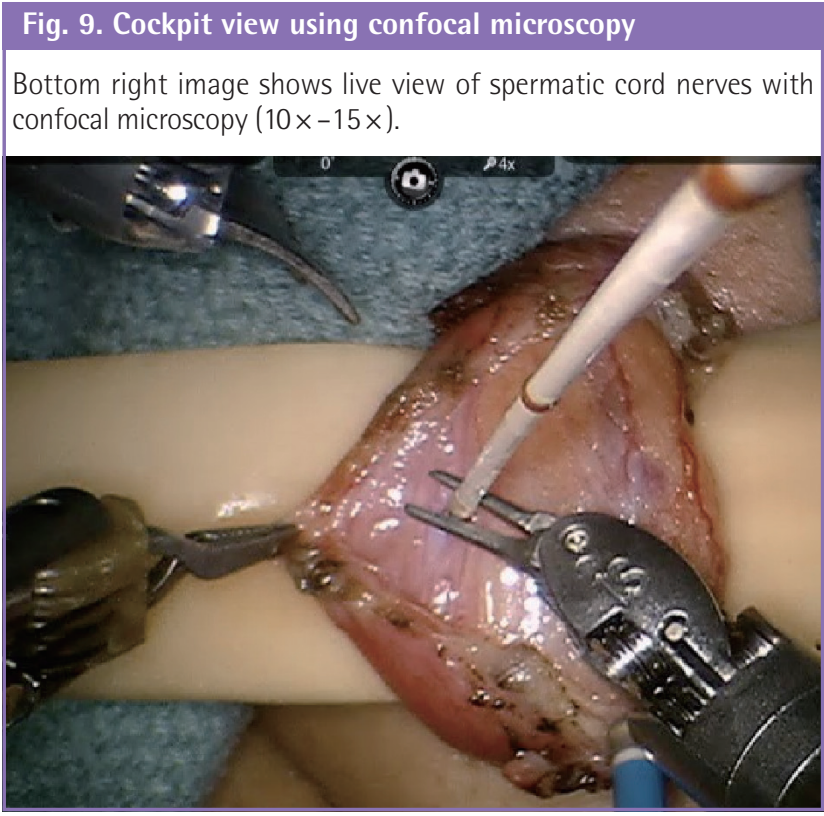

\section{Fig. 8. VeinViewer image of spermatic cord veins}

(A) View of spermatic cord and venous structures (dark) using the VeinViewer. (B) View of spermatic cord vasculature without the assistance of the VeinViewer $(10 x-12 x)$.
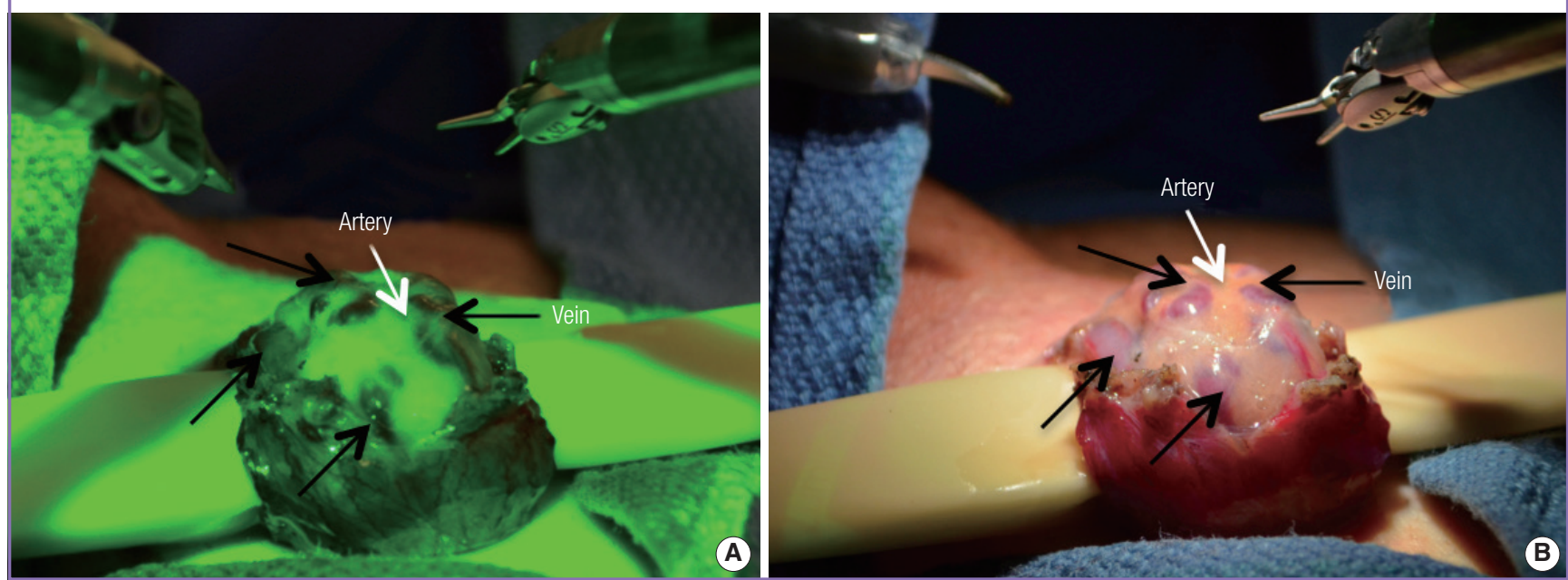
level. Confocal microscopy with Cellvizio (Mauna Kea Technologies, Paris, France) allows in vivo histology at a subcellular resolution of tissue inside the body. The small probes are compatible with all flexible video endoscopes and therefore small enough for manipulation by robotic instruments. The probe can be placed directly on the tissue of interest without risk of collateral damage (Fig. 9). Confocal microscopy requires contrast agents for fluorescence imaging such as fluorescein and acriflavine. In a recent study on the enteric nervous system, acriflavine was highly absorbed by neuronal cells, which allowed the clear identification of the enteric nervous system [14]. In the future, confocal microscopy could assist in identification of small residual nerve fibers within the spermatic cord.

\section{CONCLUSIONS}

As more microsurgeons venture into robot-assisted microsurgery, the demand for enhanced instrumentation will increase. This will likely drive the industry to develop better tools for microsurgical procedures.

\section{REFERENCES}

1. Parekattil SJ, Cohen MS. Robotic surgery in male infertility and chronic orchialgia. Curr Opin Urol 2010;20:75-9.

2. Mamelak AN, Drazin D, Shirzadi A, et al. Infratentorial supracerebellar resection of a pineal tumor using a high definition video exoscope (VITOM(R)). J Clin Neurosci 2012; 19:306-9.

3. Hyams ES, Perlmutter M, Stifelman MD. A prospective evaluation of the utility of laparoscopic Doppler technology during minimally invasive partial nephrectomy. Urology 2011; 77:617-20.

4. Cocuzza M, Pagani R, Coelho R, et al. The systematic use of intraoperative vascular Doppler ultrasound during micro- surgical subinguinal varicocelectomy improves precise identification and preservation of testicular blood supply. Fertil Steril 2010;93:2396-9.

5. Parekattil S, Moran M. Instruments. In: Liverneaux PA, Berner SH, Bednar MS, et al., editors. Telemicrosurgery: robot assisted microsurgery. Paris: Springer; 2013. p.31-42.

6. Toth S, Vajda J, Pasztor E, et al. Separation of the tumor and brain surface by "water jet" in cases of meningiomas. J Neurooncol 1987;5:117-24.

7. Menovsky T, De Ridder D. Hydrodissection versus highpressure water jet dissection. Microsurgery 2007;27:354.

8. Parekattil SJ, Gudeloglu A, Brahmbhatt JV, et al. Trifecta nerve complex: potential anatomical basis for microsurgical denervation of the spermatic cord for chronic orchialgia. J Urol 2013;190:265-70.

9. Tulikangas PK, Smith T, Falcone T, et al. Gross and histologic characteristics of laparoscopic injuries with four different energy sources. Fertil Steril 2001;75:806-10.

10. Quaas AM, Einarsson JI, Srouji S, et al. Robotic myomectomy: a review of indications and techniques. Rev Obstet Gynecol 2010;3:185-91.

11. Vincent R, Bittermann AJ, Oates J, et al. KTP versus $\mathrm{CO}_{2}$ laser fiber stapedotomy for primary otosclerosis: results of a new comparative series with the otology-neurotology database. Otol Neurotol 2012;33:928-33.

12. Strehle EM. Making the invisible visible: near-infrared spectroscopy and phlebotomy in children. Telemed J E Health 2010;16:889-93.

13. Phipps K, Modic A, O’Riordan MA, et al. A randomized trial of the Vein Viewer versus standard technique for placement of peripherally inserted central catheters (PICCs) in neonates. J Perinatol 2012;32:498-501.

14. Sumiyama K, Kiesslich R, Ohya TR, et al. In vivo imaging of enteric neuronal networks in humans using confocal laser endomicroscopy. Gastroenterology 2012;143:1152-3. 